\title{
Coping with University Students' Boredom in Classroom: A Behavioral Perspective
}

\author{
Xinhua $\mathrm{Hu}$ \\ Business School, Southwest University of Political Science and Law, \\ 301, Baosheng Road, Yubei District, Chongqing 401120, China \\ Tel: 86-189-8329-8192 E-mail: xinhua_h@126.com
}

Received: July 10, $2020 \quad$ Accepted: August 30, $2020 \quad$ Published: September 6, 2020

doi: 10.5296/ire.v8i2.17648 URL: https://doi.org/10.5296/ire.v8i2.17648

\begin{abstract}
In the digital age, influenced by various electronic devices, social networking applications and video games, boredom deepens in university classrooms. According to B. J. Fogg's Behavior Model, university students feel boring in the classroom, and difficult to carry out their learning behavior, it is because of at least one factor is absent among learning motivation, learning ability and triggering factors. University teachers should start with the above three factors and take reasonable ways to motivate students' internal and external learning motivation according to the requirements of different motivations, effectively match learning ability and learning tasks, set a reasonable task difficulty for students' learning, and design the learning tasks based on the problem solving in and out of the class involving students as the main body of learning. These measures will trigger students' enthusiasm for learning, shielding boring emotions, and implementing learning behaviors spontaneously.
\end{abstract}

Keywords: Classroom boredom, Learning motivation, Learning ability, Triggering factors

\section{Introduction}

We are living in a digital age, and digital objects, such as electronic text, sound, image, audio, video, network and other forms have created a virtual world independent of the physical world. Games, digital entertainment, online shopping, electronic payment have become an important part of life. These activities have gradually become people's understanding and practice of the world to construct people's life style. According to a 2014 survey by Millward Brown, an international market research organization, Chinese people spend the most time staring at computers and laptop screens in the world, with an average of 161 minutes, while the average time of using smart phones is 170 minutes, which is also in the forefront of the 
world. In this context, our original living experience based on various direct feelings or real existence is gradually replaced by various direct feelings or virtual exchanges of digital objects. As the presentation carrier of digital objects, the frequent use of smart phones, tablet computers, laptops and other electronic devices has brought about the consequences. As pointed out by psychologist Palladino, boredom has become an epidemic. Compared with the 24-hour non-stop media, video games and the internet, it is difficult for teachers to excite students.

Based on this, this paper attempts to explore how university teachers deal with classroom boredom and construct a strategy system to help university teachers improve students' concentration in the classroom.

\section{Literature Review}

According to APA Dictionary of psychology, boredom is defined as "a kind of tiredness or tiredness resulting from a lack of concentration on stimuli in the environment". Studies have found that when a person is in a repetitive, monotonous and depressive modern environment, he is more likely to have a tendency of boredom (Zhou et al., 2012). Smith (1981) thinks that boredom may be caused by the monotony of repetitive tasks that individuals have become accustomed to. Perkins and Hill (1985) proposed that boredom also occurs when individuals are at a low level of arousal both psychologically and cognitively. According to Pekrun (2006), boredom is a multi-dimensional emotion, including unpleasant feeling (emotion), low arousal (physiology), desire to leave boring situation (motivation) and slow perception time (cognition). Gerritsen et al. (2014) believe that inattention, hyperactivity and executive dysfunction can predict boredom. In a related experimental study, Wilson et al. (2014) exposed the subjects to a monotonous laboratory environment for 15 minutes, deprived them of all the objects and activities that could make them concentrate. It was found that some of the subjects chose to apply electric shock to themselves to avoid chatting time.

Therefore, boredom has a direct relationship with attention. Boredom occurs mainly in the following situations: (1) when we fail to successfully focus our attention on the internal (such as thinking or emotion) or external (such as environmental stimulation) information needed to participate in satisfying activities; (2) focusing on the reality that we can't concentrate and participate in satisfying activities; (3) attributing our disgusting state to the environment. In an experimental study, subjects were placed in a monotonous laboratory environment for 15 minutes, depriving them of all the objects and activities that allowed them to concentrate. It turned out that some of them chose to shock themselves to escape boredom. On the other hand, long-term boring experience will further lead to individual inattention, resulting in a variety of cognitive errors and cognitive needs decline. Seib and vodanvich (1998), Wallance et al. (2003) pointed out in the opposite direction that long-term boring experience will lead to individual inattention, resulting in various cognitive errors and cognitive needs decline. Wang (2014) conducted a study on university students, and the results showed that there was a significant negative correlation between mindfulness and boredom. Mindfulness is a self-regulation method for individuals to consciously maintain their attention in the present without any judgment, which can effectively improve the individual's attention level. 
Learning boredom is a field with rich literatures in the study of boredom. For example, Zhao and Zhang (2015) conducted a questionnaire survey on university students, which subdivided the boredom tendency shown by different types of universities and different grades of university students. Mihaly Csikszentmihalyi (1990) believes that when an individual's skill exceeds the difficulty of a task, he will experience the boredom of a task; when the difficulty of a task exceeds the skill of an individual, he will experience anxiety. Only when the difficulty of the task matches with the skill of the individual, can the individual experience pleasure, enjoyment and interest, and may be absorbed in the task, thus resulting in "flow" state. Pekrun et al. (2002) also pointed out that when students perceived that the task requirements were too low or too high, or that they could not perceive the value from the academic tasks, they would be bored. Schukajlow and Rakoczy (2016) studied students' emotion as an intervention variable between the premise and result of teaching method, motivation and performance. The research results show that students who can expand more solutions enjoy mathematics class more and experience less boredom, and enjoy learning process plays an intermediary role in promoting them to explore more solutions and interests, it plays a mediating role between pre-test interest and post-test interest.

In general, boredom in university classroom is a common phenomenon, which is influenced by many factors. If we can't suit the remedy to the case and adapt teaching methods to improve students' interest in learning, this kind of boredom will be further deepened and students will lose their expectation of class. If we say that in the past years, university teachers can not worry too much about the boredom of university students' classroom. In the digital age, this kind of boring tendency will pose unprecedented challenges to university teachers, which has become a problem that must be paid attention to.

\section{University Students' Classroom Boredom in the Digital Age}

The boredom of university students in the classroom has never been absent, but in the digital age, this sense of boredom has been significantly deepened. The popularity of electronic devices, especially smart phones, has brought a significant impact on university classrooms. According to a survey by a Chinese university, more than $90 \%$ of the students have used mobile phones in classroom, and most of them are for entertainment rather than learning. Students are already accustomed to fragmentation news, videos and games. Even if they have only a few minutes to break, they will also use their mobile phone to see WeChat's friends circle, microblog, Tik Tok or news. Once the brain adapts to this kind of fast or real-time feedback behavior, the attention focusing time becomes shorter and shorter, and likes multi task switching. When learning or working with low stimulation / boring, it is unable to effectively focus on attention, and those long and unattractive speeches are not attractive classes and even books have been difficult to stimulate students' brains.

On the other hand, when a large amount of professional information is presented on the network, especially the characteristics of easy access to knowledge and autonomous learning of social sciences, students and teachers are at the same level in information contact, so that students have already known about a lot of knowledge and cases that teachers have said, or search the Internet on the spot in the classroom, if teachers cannot provide new content or 
analysis, they are facing difficulties in arousing students' interest. In recent years, the rapid development of online classroom, represented by MOOC courses and Ted lectures, as an important part of digital materials, has greatly expanded the channels and selection range of university students' knowledge learning. In order to attract learners and improve the sustainability of learning, they have strengthened learning interest, closely combined with life and work, and constructed auxiliary community platform, which enhance the requirements of university students for the process of knowledge learning. Meanwhile, the classroom of university teachers, compared with students and online classroom, is boring and lack of passion. Therefore, the current university classroom is more difficult to grasp, more difficult to convince students, but also more likely to lead to students' boring tendency.

\section{The Behavior Mechanism Behind University Students' Classroom Boredom}

If we take focused learning as a behavior of university students, the classroom boredom makes it difficult to achieve focused learning. Accordingly, to change its boring state, we should try to make them focus on classroom learning.

B. J. Fogg, professor of Stanford University, pointed out that the change of human behavior is formed by the combination of motivation, ability and triggers. If there is no motivation, there is no willingness to change behavior; if there is no corresponding ability to change behavior, it means that the cost of changing behavior is too high, and there will be no behavior change. When motivation and ability are both available, timely warning is also needed to enable the target object to enter the task of behavior change. University students show boredom in class, that is, they do not carry out learning behavior, there are at least one of three elements is a lack.

\subsection{Lack of Learning Motivation}

The motivation of learning comes from the internal and external fields. The internal motivation can be considered to be formed by the desire for knowledge exploration, and the external motivation is formed by coping with the examination, peer identification or future career recognition. In the internal motivation, learners' needs, interests and emotions have an important impact, mainly involving curiosity, interest in the activity itself, enjoyment, competence, achievement needs and so on. External motivation mainly comes from social identity in learning, which is reflected in the respect needs and social needs in Maslow's hierarchy of needs theory. In group learning, respect, competition and cooperation are the group psychological factors that stimulate and maintain learners' learning motivation. In reality, the lack of clear learning objectives or ideals, the feeling that reading is useless or the perception of learning value is low, and the lack of interest in learning knowledge and other factors constitute the main causes of the lack of internal motivation of University Students' classroom learning. Fear of failure, lack of interaction with classmates and teachers, and the influence of small group learning atmosphere constitute the main causes of lack of external motivation.

\subsection{Learning Ability and Tasks do Not Match}

University students do not implement learning behavior in the ability dimension, not 
necessarily because of the difficulty of learning task is too high, more likely is the difficulty of learning task and ability does not match, too high or too low task requirements will produce boredom. One reason is that the learning task is too difficult. Some university students follow the learning methods of high school and do not master the professional knowledge learning methods of University. As a result, the learning progress can not keep up with the teaching progress of teachers. Once a course falls behind, the learning of the whole course will become very passive. As a result, more and more students have no confidence, that is, the sense of learning efficacy is getting lower and lower, and it is more and more difficult to enter the learning state in the classroom. Second, when the difficulty of learning task is too low, students will not feel challenging, and they will think that whether there is classroom learning has little impact on learning effect, and it will also lead to difficulty in focusing in class.

\subsection{The Trigger Factor Is Absent}

Under the condition of both learning ability and learning motivation, some university students are still bored in the classroom. The reason lies in the lack of boosting power to push students into learning state. In reality, teachers and counselors often use the form of putting facts and reasoning to stimulate students' learning enthusiasm, but the effect is not obvious. Because these principles and facts are not unfamiliar to them, they can not be internalized into a spark to ignite their enthusiasm for learning. A large number of external factors, especially the strong temptation of various social software and news media on mobile phones, have significantly reduced students' learning enthusiasm. One study found that undergraduates who spend more than three hours a day online have significantly higher boredom than those who spend less than or equal to three hours a day online.

\section{Strategies to Change University Students' Classroom Boredom}

In order to promote university students to get rid of boredom and put their body and mind into study, teachers should start from the three elements of motivation, ability and trigger factors according to the actual situation, not only to enhance the single role of each element, but also to consider the three elements closely linked into a whole, to jointly promote university students to eliminate classroom boredom and actively participate in classroom learning.

\subsection{Improve Learning Motivation}

The promotion of university students' learning motivation also needs to start from internal motivation and external motivation. Moreover, the focus of the cultivation and promotion of learning motivation is more outside the classroom. From the perspective of internal motivation, teachers should strive to establish the sense of goal and value of university students' learning, improve their recognition of their major and curriculum knowledge, and then enhance their interest in learning, so as to obtain a sense of pleasure in learning. From the perspective of external motivation, the key is to create a good learning atmosphere, develop students' internal learning desire and enhance their learning enthusiasm by mutual influence, competition and cooperative learning among students. 
University counselors have more opportunities and channels of emotional communication with university students than other teachers, so they have incomparable advantages in promoting students' motivation. First of all, university counselors need to communicate with students to make students realize the role of university classroom learning in university life and even the whole life, integrate resources inside and outside the school, help students plan their future goals, sort out the connection between learning and goals, so as to stimulate students' deep-level internal learning motivation. Secondly, university counselors should focus on creating a good learning atmosphere, creating a benign class culture and dormitory culture that is conducive to promoting learning, so that students can not only have a healthy competition to drive them to study hard, but also actively cooperate and help each other to grow and progress together, so as to make learning performance, class and dormitory culture become important elements of mutual recognition among students.

The key role of teachers is to enhance students' interest in the curriculum and value cognition. Therefore, on one hand, teachers need to combine their courses closely with the students' future professional development direction, deeply understand the position of the curriculum knowledge in the students' overall learning system, as well as the value that can be brought in the students' future career. This needs to improve the teachers' systematic thinking and avoid only understanding the content of a single knowledge unit of the course, but not learning clearly Students' overall learning system. On the other hand, teachers need to constantly improve their teaching ability, explore the best teaching methods suitable for the courses they teach, such as case teaching method, project-based teaching method, mixed teaching method, flipped classroom, etc., emphasizing that students can realize deep learning from mechanical memory of knowledge to deep understanding and then to transfer application. And integrate online and offline resources, combine the teaching knowledge with the most cutting-edge trend or trend of students' concern, dynamically update the teaching content, so as to stimulate students' internal and external learning motivation.

\subsection{Match Learning Ability With Learning Task}

To match learning ability with learning task, teachers need to improve students' learning ability and arrange the difficulty of learning task reasonably.

Firstly, to improve learning ability. The key point is to pay special attention to the students with low learning ability and create a class atmosphere of mutual help and mutual help. When some students encounter difficulties in learning, they will help each other to provide support and encouragement for them or her to overcome the learning difficulties, so as to reduce the negative factors such as anxiety, anxiety and fear that affect motivation, which is conducive to stimulate their learning motivation and promote their growth together. On the other hand, for the students who provide help for students with learning difficulties, they will have a deeper sense of satisfaction if they use their knowledge and skills to help them to participate in learning activities in a better state.

Secondly, reasonably arrange learning task difficulty. The key point is to dynamically grasp the degree of students' mastery of curriculum knowledge. The tasks arranged should not only be challenging, but also be able to achieve the goal after their efforts. On one hand, teachers 
can get the students' knowledge base by asking them questions or using quizzes in classes, and adjust the content of lectures or the difficulty of students' homework from easy to difficult; for students who already have certain basic knowledge, teachers should set up novel learning tasks to provide students with different perspectives. On the other hand, teachers and students are required to strengthen the communication in and outside the class, keep abreast of the students' learning progress, and urge the students to preview before class and review after class to consolidate the knowledge learned, so as to lay a foundation for the matching of new lecture content and students' knowledge base.

Thirdly, to use gamification method for learning of difficult knowledge. Because the current university students are growing up with digital games, the tasks set in the game ensure that they are attractive to players. The key point lies in the challenging task setting and timely feedback, which can make players feel the pleasure of successful breakthrough. The classroom teaching can learn from the concept of game design, find out the difficult problems of students through targeted exercises, and compile the learning process of high difficulty knowledge into the form of game activities, so as to ease students' fear of difficulties and improve their interest. In addition, feedback and summary can be given in combination with students' classroom performance to urge students to further think about knowledge and learning process, consolidate knowledge, improve thinking methods and improve learning ability.

\subsection{Effective Triggering}

The key to trigger university students' enthusiasm for learning in the classroom and encourage them to actively participate is to construct the sense of meaning and value of students' learning. It's important to help students establish learning goals according to their learning value needs. University teachers can use Maslow's "hierarchy of needs theory" to explore students' basic learning needs and high-level needs. The high-level needs usually not only play a role in the current learning situation, but also play an important role in students' career orientation and development goals in their future life. Therefore, through having an insight into the high-level needs of students, teachers can integrate them with the current situation, to stimulate the enthusiasm of students with the combination of learning.

Problem solving is the source of learning sense of meaning. The task of solving problems should be carefully designed in the classroom, and the knowledge learned should be closely combined with solving specific problems in reality. The real situation originated from real life or social needs should be constructed. Students should be introduced into it in the form of a team. Students' autonomous learning ability, knowledge expansion ability, creativity and cooperation ability should be brought into full play to make them become knowledge application and creation the main body, through creating a good environment and atmosphere and timely and effective guidance, helps students produce innovative and unique ideas in the process of finding, analyzing and solving problems. In addition to the tasks in class, it is also necessary to ask students to experience the real practice around the knowledge points they have learned, feel what they are doing and how to do in practice, deepen the understanding and thinking of knowledge points through practical experience, and further understand the 
application of knowledge points in reality, and the relationship and connection path with other relevant knowledge points. Students are required to share their extracurricular experience in class and combine it with the classroom task, and the situation that students are familiar is added to the task to strengthen the realistic sense of problem-solving.

In the process of problem solving, teachers should consciously introduce the modern digital equipment owned by students into it, and make full use of the convenient communication mode and rich resources provided by social application software and network teaching platform to improve the efficiency of problem solving. This can not only meet the psychological needs of students who are inseparable from electronic devices, especially smart phones, but also provide favorable conditions for cooperation between students, communication between teachers and students in and out of class, network resource acquisition and timely feedback.

\section{Acknowledgement}

The research is financed by The Humanities and Social Sciences Research Project of Chongqing Education Commission, approval No.: 20SKSZ010; The Higher Education Teaching Reform Project of Chongqing Education Commission, approval No.: 153042; The Teaching Reform Project of Southwest University of Political Science and Law's, approval No.: 2015C02; The Ideological and Political Education Research Project of Southwest University of Political Science and Law's, approval No.: 2019-XZSZ01.

\section{References}

Csikszentmihalyi, M. (1990). Flow: The Psychology of Optimal Experience. New York, NY: Harper \& Row, Publishers.

Dong, W., Li, Z., \& Li, X. (2018). The relationship between boredom and well-being of university students: The mediating role of internet dependence. Chinese Journal of Clinical Psychology, 26(5), 1034-1037.

Eastwood, J. D., Frischen, A., \& Fenske, M. J. (2012). The unengaged mind: Defining boredom in terms of attention. Perspectives on Psychological Science, 7(5), 482-495. https://doi.org/10.1177/1745691612456044

Fogg, B. J. (2009). A behavior model for persuasive design. Persuasive '09: Proceedings of the 4th International Conference on Persuasive Technology, April 2009. https://doi.org/10.1145/1541948.1541999

Gerritsen, C. J., Toplak, M. E., \& Sciaraffa, J. (2014). I can't get no satisfaction: Potential causes of boredom. Consciousness \& Cognition, 27, 27-41. https://doi.org/10.1016/j.concog. 2013.10.001

Huang, S., Li, D., \& Zhang W. (2010). Preliminary development of university students' boredom tendency questionnaire. Psychological Development and Education, 26(3), 308-314.

Liu, Y. (2017). The nature and future of digital age. China Social Sciences Journal. 
Pekrun, R. (2006). The control-value theory of achievement emotions: Assumptions, corollaries, and implications for educational research and practice. Educational Psychology Review, 18, 315-341. https://doi.org/10.1007/s10648-006-9029-9

Pekrun, R., Goetz, T., \& Titz, W. (2002). Academic emotions in students' self-regulated learning and achievement: A program of qualitative and quantitative research. Educational Psychologist, 37(2), 91-105. https://doi.org/10.1207/S15326985EP3702_4

Perkins, R. E., \& Hill, A. B. (1985). Cognitive and affective aspects of boredom. British Journal of Psychology, 76(2), 221-234. https://doi.org/10.1111/j.2044-8295.1985.tb01946.x

Ren, Y., \& Zhu, G. (2009). Meaningful learning comes from problem solving: An interview with professor David Jonathan. China Education Technology Journal, 1, 6-10.

Schukajlow, S., \& Rakoczy, K. (2016). The power of emotions: Can enjoyment and boredom explain the impact of individual preconditions and teaching methods on interest and performance in mathematics? Learning \& Instruction, 44, 117-127. https://doi.org/10.1016/ j.learninstruc.2016.05.001

Seib, H. M., \& Vodanovich, S. J. (1998). Cognitive correlates of boredom proneness: The role of private self-consciousness and absorption. Journal of Psychology, 132(6), 642-652. https://doi.org/10.1080/00223989809599295

Smith, R. P. (1981). Boredom: A review. Human Factors, 23, 329-340. https://doi.org/10.11 77/001872088102300308

Tan, S. (2019). Construction of "blended teaching" mode for deep learning in universities. China Higher Education, 6, 51-53.

Wallace, J. C., Vodanovich, S. J., \& Restino, B. M. (2003). Predicting cognitive failures from boredom proneness and daytime sleepiness scores: An investigation within military and undergraduate samples. Personality \& Individual Differences, 34(4), 635-644. https://doi.org/10.1016/S0191-8869(02)00050-8

Wang, Z., Li, X., \& Lin, J. (2015). Mobile phone and Internet plus class: new thinking and new path of integrating information technology with teaching. Journal of Distance Education, $4,14-21$.

Wilson, T. D., Reinhard, D. A., \& Westgate, E. C. (2014). Just think: The challenges of the disengaged mind. Science, 345(6192), 75-77. https://doi.org/10.1126/science.1250830

Zhang, Y., Li, S., \& Yu, G. (2019). The relationship between boredom and cognitive failure in university students: The mediating role of mobile phone addiction tendency and its difference between the only child group and the non-only child group. Psychological Development and Education, 35(3), 344-351.

Zhou, H., Wang, Q., \& Dong, Y. (2012). Boredom: a long-standing and emerging research topic. Progress in Psychological Science, 20(1), 98-107. https://doi.org/10.3724/SP.J.1042. 2012.00098 


\section{Copyright Disclaimer}

Copyright reserved by the authors.

This article is an open-access article distributed under the terms and conditions of the Creative Commons Attribution license (http://creativecommons.org/licenses/by/4.0/). 